\title{
Nuclear fusion and the helium supply problem
}

\author{
A. M. Bradshaw ${ }^{\mathrm{a}}$, T. Hamacher ${ }^{\mathrm{b}}$ \\ ${ }^{a}$ Max Planck Institute for Plasma Physics, Association IPP-Euratom, Boltzmannstr. 2, 85741 Garching, \\ and Fritz Haber Institute of the Max Planck Society, Faradayweg 4-6, 14195 Berlin, Germany \\ ${ }^{\mathrm{b}}$ Institute for Energy Economy and Application Technology, Technical University of Munich, Arcisstrasse 21, \\ 80333 München, Germany
}

\begin{abstract}
The natural resources required for the operation of fusion power plants are - with the possible exception of the neutron multiplier beryllium - readily available. On the other hand, the supply of helium, which is required as cryogenic medium and coolant, may be a problem due to losses during operation and decommissioning. Helium is a rare element obtained as a by-product in the extraction of natural gas. The danger exists that the natural gas will be used up without the helium being conserved. We estimate the helium demand for a global $30 \%$ base-load contribution of fusion to electricity supply and also calculate the amount produced by the power plants themselves.
\end{abstract}

Keywords: helium, fusion power plant, DEMO, sustainability, cryogenic system, blanket

\section{Introduction}

Unless there are substantial advances in the technology for the manufacture of high temperature superconducting cable in the nest few years, we can assume that helium-based cryogenic systems will be required for the first fusion power plants. Helium is also a strong candidate for coolant gas in the blanket, although there are other possible solutions. Although helium is the second most abundant element in the universe, the average concentration in the Earth's crust is probably not even in the ppb range, and in the Earth's atmosphere it is only $5.2 \mathrm{ppm}$ per volume. Fortunately, helium has concentrated in certain regions of the crust, in particular in natural gas deposits, and can be readily extracted. At the time of writing, however, there is a shortage of helium, with concomitant rising prices [1]. Does this mean that helium resources are already being depleted at such a rate that exhaustion is looming? Could this affect the development of fusion power with its high demand for helium, both as cryogenic gas and as a coolant? Not only are there technical and economic aspects to this discussion, but also ethical questions to be answered. The fusion community often describes fusion power as "sustainable" in the ecological sense of the word. The rapid consumption of helium from natural gas deposits, which has accumulated over billions of years, leaving little or nothing for future generations, is hardly defensible and certainly not sustainable. Cook was one of the first to present this "conservationist" view on helium (the word "sustainability" was not yet in use) [2]. In the present paper, we re-examine this question in the spirit of our recent discussion on sustainability with respect to the materials required for the generation, transmission and storage of renewable energy [3].

In Ref. [4] the availability of the fusion fuels lithium and deuterium, as well as of the neutron multiplier materials beryllium and lead, was described. It was our original intention to include helium in that paper, but mention was excluded because of space restrictions. An extremely useful book on many aspects of the helium problem, edited by Nuttall et al. [5], appeared just as the present paper was being drafted. Moreover, in a recent journal article [6], Nuttall et al. have justifiably criticised the way our society squanders helium. The book also contains an article on helium and fusion energy by Clarke and Cai [7], with whom we generally concur.

\section{Terrestrial helium: origin and occurrence}

\subsection{Helium in the Earth's crust and atmosphere}

The majority (probably about 95\%) of terrestrial helium derives from the nuclear decay of uranium and thorium in the Earth's crust; the rest is of stellar origin. The main contributors are the isotopes ${ }^{238} \mathrm{U}$ and ${ }^{232} \mathrm{Th}$, which decay in chain reactions via $\alpha$ and $\beta$ emission to produce stable isotopes of lead. Most of the helium thus created has diffused to the surface and escaped into the atmosphere over the last 4 billion years, but a small fraction thereof has been trapped by impermeable layers of rock. Natural gas, consisting mainly of methane, also collects in such geological constellations, so that helium for commercial use is normally produced from natural gas, where it is a minor component with a concentration up to, but very rarely exceeding, about one per cent.

Although it was already clear in the 1920's that the helium was of radiogenic origin, a problem later became apparent. Several authors pointed out that, based on the amount of uranium and thorium in the Earth's crust $(\approx 1$ $\mathrm{x} 10^{14} \mathrm{t}$ and $5 \times 10^{14} \mathrm{t}$, respectively) and the half-lives of the two parent nuclei, the rate of generation of helium is about $3 \times 10^{3}$ t per year, which also corresponds to the rate of degassing, i.e. the rate of emission into the atmosphere [8]. Moreover, about $1 \times 10^{14} \mathrm{t}$ helium has passed into the Earth's atmosphere since the formation of the planet. Yet the concentration of helium in the Earth's atmosphere is only $5.2 \mathrm{ppm}$ (by volume) at sea level, corresponding to a total of $3.5 \times 10^{9} \mathrm{t}$, and is presumed to have remained more or less constant on the 
geological time scale! This means that about $10^{14} \mathrm{t}$ must also have been lost from the upper atmosphere during this period. The high altitude ionosphere consists of an almost collisionless plasma containing $\mathrm{H}^{+}, \mathrm{He}^{+}$and $\mathrm{O}^{+}$ ions. The latter move along the Earth's magnetic field lines, which normally remain closed. However, the interaction with the solar wind above the poles causes magnetic reconnection to take place, so that the field lines open and the plasma flows into the magnetosphere and eventually into interplanetary space. The effect was predicted by Axford [10], who termed it the "polar wind". There are probably other outflow mechanisms, but the polar wind is thought to be the most important for helium [11].

To conclude this section we note, firstly, that it is indeed only a very small fraction, less than $10^{-7}$, of the total amount of the radiogenic helium formed in the Earth's crust in the last four billion years which has accumulated, fortuitously and fortunately, in appropriate geological strata. Despite this huge amount of helium that has passed through the Earth's atmosphere, the annual rate of production in the crust is only about $3 \mathrm{x}$ $10^{3} \mathrm{t}$ per year. Secondly, there is no prospect of tapping into this supply, because of the high degree of dispersion of the sources, i.e. of the radioactive nuclei in various uranium and thorium minerals.

\subsection{Helium reserves and resources}

As shown in Table 1, the total helium reserves and resources in the United States - as of the year 2006 - are given by the United States Geological Survey (USGS) [11] in metric tons as $3.6 \times 10^{6} \mathrm{t}$ and the total resources outside the US as $5.2 \times 10^{6} \mathrm{t}$, thus giving a global value of $8.8 \times 10^{6} \mathrm{t}$. (Note that this is a factor of several hundred less than the amount of helium in the atmosphere.) The USGS defines "resources" as deposits of the solid, liquid or gaseous material in or on the Earth's crust in such a condition that economic extraction is potentially feasible. Resources can be further divided into "identified" and "undiscovered". "Reserves" are deposits which can be exploited economically at the present time.

From Table 1 it is clear that - after the US - Qatar, Algeria and Russia have the largest resources at their disposal. It is therefore hardly surprising that new plants in conjunction with LNG production will shortly come online in Algeria (Arzew 2) and Qatar (Qatar 2). Moreover, and importantly, Russia is contemplating - in addition to Orenberg, where the He concentration is apparently very low at well under $0.1 \%$ - opening up the Kovykta and Chayanda areas for production [12]. "Others" includes Australia, where a new plant in Darwin has just gone online. Nineteen plants are currently in operation in the US, as well as 7 elsewhere.

The large unknown in such quantitative assessments is always the extent of the undiscovered resources. Of the US resources given some are designated "probable" $\left(9.3 \times 10^{5} \mathrm{t}\right)$, "possible" $\left(9.0 \times 10^{5} \mathrm{t}\right)$ and "speculative" $\left(9.9 \times 10^{5} \mathrm{t}\right)$. It is not clear whether the latter belong to the undiscovered resources. Details of this sort are not available for helium resources outside the US. We note at this point that shale gas contains only very small amounts of helium, invariably under the $0.04 \%$ barrier normally regarded as economical for extraction ${ }^{1}$.

Table 1: Global helium reserves and resources according to the USGS, 2012 [11]; na = not available; the US reserves include the helium in the Cliffside Field federal reserve facility.

\begin{tabular}{|l|c|c|}
\hline & $\begin{array}{c}\text { Resources } \\
\left(10^{6} \mathrm{t}\right)\end{array}$ & $\begin{array}{c}\text { Reserves } \\
\left(10^{6} \mathrm{t}\right)\end{array}$ \\
\hline US & 3.48 & 0.72 \\
Qatar & 1.70 & na \\
Algeria & 1.39 & 0.30 \\
Russia & 1.15 & 0.29 \\
Canada & 0.34 & na \\
China & 0.19 & na \\
Others & 0.52 & na \\
Total & $\mathbf{8 . 7 7}$ & \\
\hline
\end{tabular}

Could the extraction of helium from air (5.2 ppm) be a "back-stop" technology in the sense of the debate on unlimited substitutability (see e. g. Ayres [13]) in the 1970's? This problem has been treated by Krupka and Hammel [14] who show that the ideal extraction energy of helium from air is $\approx 2.3 \mathrm{MWh} \mathrm{t}^{-1}$. Comparison with a simple empirical model based on actual extraction energies gives a value four orders of magnitude higher, i.e. $\approx 20 \mathrm{GWh} \mathrm{t}^{-1}$ ! The authors attribute the very low efficiency to the large mechanical and thermal energy losses associated with the massive amounts of gas that would be passing through the separation plant. Clarke and Clare, in their treatment of the problem [15], note that over a hundred cubic kilometres of air would have to be processed per day in order to satisfy the present global helium demand. New uses would also have to be found for the other cryogenic fluids obtained as byproducts. Oxygen, for example, could be used for the oxy-fuel process in conjunction with carbon capture and storage. New membrane technology [16] could perhaps improve the separation efficiency. The $5.2 \mathrm{ppm}$ helium in the atmosphere clearly have back-stop potential.

\subsection{Other uses of helium and the present helium supply problem}

As noted above, global production of helium was 1.8 x $10^{8} \mathrm{~m}^{3}$, or $3.1 \times 10^{4} \mathrm{t}$, in $2011 ; 78 \%$ of this was produced in the United States [11]. Reliable figures for usage are only available for the US, but are not expected to be vastly different globally. For the year 2011 the

\footnotetext{
${ }^{1}$ When helium is extracted during the primary process of collecting and purifying (gaseous) methane, as in the plants in the US, a minimum $0.3 \%$ helium concentration in the feed gas is regarded as economically feasible. The $0.04 \%$ minimum concentration applies to natural gas, which is converted to LNG. The helium is extracted from the tail gases [17].
} 
figures were: Cryogenics (in particular magnetic resonance imaging, but also physics research) $32 \%$, pressurising and purging $18 \%$, welding (as protective gas) $13 \%$, leak detection $4 \%$, breathing mixtures $2 \%$ and others (including party balloons!) $13 \%$ [17].

Helium supply in the last few years has been characterised by shortages and price rises [1]. The latter, however, have been moderate (2012: ca. $25 \%$ for crude helium in comparison to 2009 , despite the pick-up of the world economy), largely because there is not a free market in helium. The price is essentially determined by the price at which the US Bureau of Land Management sells off the helium from the federal reserve in Cliffside Field, Texas. This procedure is described by Theiss [18]. In the next few years, the supply situation will undoubtedly change due to the new helium production facilities outside the US described above, but also - in the opposite sense - by the recommendation of the NAS in the 2010 report not to run down the federal reserve as originally envisaged by the US Congress in 1996. It suffices here to note that there is at present no (geological) shortage of helium causing (economic) scarcity, but that the quantity of helium stored in natural gas deposits is finite and that the exponential reserve index (see Section 4) is less than a 100 years.

\section{The future fusion power station}

Fusion power plants are likely to be prolific users of helium. On the assumption that the magnetic field coils will use a conventional niobium-based superconductor, helium will be required for the cryogenic system. Moreover, because of its high thermal conductivity, high specific heat and inertness helium at high pressure is an ideal coolant, or energy transfer medium, in the power conversion system (PCS) of the reactor. Other solutions, however, such as water or Li self-cooling, have also been discussed. Future helium power plants will also produce helium, both in the fusion reaction itself and through the action of the neutron multiplier. In order to estimate helium inventories (loadings) and the helium production rate, we use design data from ITER [19], from the European DEMO power plant [20] (Table 2) and from the European test blanket project [21].

Table 2: Main parameters of the DEMO power reactor [20]

\begin{tabular}{|l|c|}
\hline Parameter & \\
\hline Major radius [m] & 7.5 \\
Minor radius [m] & 2.5 \\
Plasma elongation & 1.9 \\
Plasma triangularity & 0.47 \\
Source peaking factor & 1.7 \\
\hline Fusion power [MW] & 2358 \\
Net electrical power [MW] & 1000 \\
\hline
\end{tabular}

To achieve tritium self-sufficiency in a future fusion power plant, it will be necessary to employ a neutron multiplier, such as beryllium or lead, which produce further neutrons via $(\mathrm{n}, 2 \mathrm{n})$ reactions in the blanket of the reactor. In the DEMO reactor study [21] a power plant is described which uses helium coolant for two possible types of breeding blanket (HCLL and HCPB). The main DEMO parameters are given in Table 2. According to Sedano [22] and Gastaldi et al [25] as well as Boccaccini and Franza [26] the helium circuitry in the PCS will contain about $23 \mathrm{t}$ helium; this figure is approximately the same for both blankets. The power load is divided up between blanket $(85 \%)$ and divertor $(15 \%)$.

According to Serio [19] the ITER cryogenic system requires $24 \mathrm{t}$ helium; the total amount of helium on site will, on average, be about $27 \mathrm{t}$. The size of the helium inventory is primarily related to the cooling of the superconducting magnets and in particular to the length and diameter of the required circuitry. DEMO will be a larger machine with approximately $15 \%$ larger linear dimensions. The helium inventory is estimated to be $31 \mathrm{t}$ [19]. Assuming the same sort of infrastructure, the total on-site helium will be about $34 \mathrm{t}$. (Note that the increase in fusion power $-2.4 \mathrm{GW}$ as opposed to $0.5 \mathrm{GW}$ for ITER - is taken up by the PCS.) In total, the helium inventory of the DEMO power plant is about $60 \mathrm{t}$.

Extremely important for this paper is an estimate of the unavoidable losses, i.e. the amount of helium, which for a variety of reasons is not, or cannot be, recovered and is vented yearly into the atmosphere from a future fusion power plant. The annual losses of the complete cryogenic system of the LHC accelerator at CERN (helium inventory $140 \mathrm{t}$ ) were initially about $25 \%$ [20]. It is expected that a figure of this kind will also apply in the case of ITER, at least in the first year or so. After several years of operation, however, this could be reduced to about $10 \%$ [19]. Are these figures likely to pertain for the cryogenic systems of fusion power plants? Serio is of the opinion that $1 \%$ per annum would be possible in principle, if there were corresponding advances in cryo-technology specifically aimed at reducing losses and, in particular, if provision were made for collection and storage, when shutdowns of the whole plant or larger parts thereof are planned. The same applies, of course, to end-of-life decommissioning of the whole plant. In view of the present uncertainty attached to these figures, it is probably best to assume a worstcase scenario of $5 \%$ annual losses for the cryo-system in a future power plant. We can assume a lower value for the coolant, since the PCS is a closed, high-pressure system. Berton [25] gives a figure of $310 \mathrm{~kg}$ p.a., corresponding to $1.4 \%$. We take a worse case of $2 \%$. In total we then have $2.2 \mathrm{t}$ p.a. helium losses per power plant, emphasising again that the helium is recovered on end-of-life decommissioning.

Finally, we look in this section at the quantity of helium produced by the fusion reaction and, in the case of the HCPB, by the neutron multiplier. According to our earlier paper [4], the annual burn up of ${ }^{6} \mathrm{Li}$ in the HCPB and HCLL reactors is $285 \mathrm{~kg}$ and $292 \mathrm{~kg}$, 
respectively. Taking $290 \mathrm{~kg}$ p.a. for both cases, we obtain a yield of $386 \mathrm{~kg} \mathrm{He}$ p.a., remembering that for each ${ }^{6} \mathrm{Li}$ atom consumed altogether two $\alpha$-particles are produced via ${ }^{6} \operatorname{Li}(n, \alpha) \mathrm{T}$ and the fusion reaction itself. In the HCBP reactor with beryllium as multiplier there are two additional reactions producing helium: ${ }^{9} \mathrm{Be}(\mathrm{n}$, $2 \mathrm{n})^{4} \mathrm{He}$ and ${ }^{9} \mathrm{Be}(\mathrm{n}, \alpha)^{6} \mathrm{Li}$. On account of the relative cross-sections we consider only the first. The Be burn-up is [4] $190 \mathrm{~kg}$ p.a., producing $169 \mathrm{~kg} \mathrm{He} \mathrm{p.a.} \mathrm{Thus,} 555$ $\mathrm{kg}$, or ca. $0.56 \mathrm{t}$, are produced annually in the HCPB, which is about $1 \%$ of the total helium inventory of $60 \mathrm{t}$. In the case of the HCLL reactor with lead as multiplier, various $\mathrm{Pb}$ isotopes are produced in the $(n, 2 n)$ reaction [26], and there is no $\alpha$-particle emission. The amount of helium produced annually remains at $0.39 \mathrm{t}$.

\section{Sustainability criteria}

We first compare the present global production of 3.1 $\mathrm{x} 10^{4} \mathrm{t}$ p.a. with the size of the identified global resources $\left(8.5 \times 10^{6} \mathrm{t}\right)$. This ratio gives a static reserve index of 270 years, or an exponential reserve index of less than 100 years, depending on the average annual increase in production. What difference would fusion make? We use the scenario of Ref. [4]: 2,760 power plants (similar to DEMO HCPB or HCLL), which would account globally for a $30 \%$ base-load $(24,000 \mathrm{TWh})$. Using the data in Section 3 , the total helium inventory of all power plants is $1.7 \times 10^{5} \mathrm{t}$, which is $2 \%$ of identified global resources. Annual losses would be $6.1 \times 10^{3} \mathrm{t}$, decreasing the static reserve index to 230 years. Fusion as a non-sustainable consumer of helium is thus seen to exacerbate an already critical situation. On the positive side, almost $30 \%$ of the putative annual losses $(\approx 2.2 \mathrm{t}$ per plant) could be compensated by the helium produced in the power plant itself $(\approx 0.6 \mathrm{t}$ per plant $)$, if a beryllium multiplier is used. In situ helium production is an advantage of fusion over possible future high-temperature fission reactors which may also require helium as coolant.

For minerals in general, advanced prospecting methods and higher efficiency of extraction allow resources to be re-classified over time as reserves and, similarly, undiscovered resources to be re-classified as identified resources. Complete exhaustion of a particular mineral will never occur, only an "effective" exhaustion, where the cost of producing a further tonne in terms of energy, water and environmental damage will be so great, that mining activities will cease. Moreover, a substitute would be found. Helium, however, is not a normal mineral commodity. Firstly, there is no substitute for its most important application, namely, as a cryogenic medium. Secondly, "effective" exhaustion of helium in the Earth's crust would mean having to extract helium from the air, which in turn would require considerably more energy than extraction from natural gas (see below). Effective exhaustion of crustal helium will occur anyway because of mankind's thirst for "cheap" fossil fuel: natural gas will be used up in the next 1-2 centuries without the helium being extracted and conserved for future generations.
How much energy would actually be required, if helium had to be extracted from the atmosphere? Using the empirical relation in Ref [14], the energy required to replace the net loss of $1.6 \mathrm{t}$ per annum ( $2.2 \mathrm{t}$ less $0.6 \mathrm{t})$ of helium per power plant would be about $45 \mathrm{GWh}$ p. a. This corresponds to only about $1 \%$ of its annual output! Clarke and Clare [15] give a similar value, but also point to the problems associated with processing such large volumes of air. We conclude that, even in a situation where helium has to be extracted from air, fusion would remain a sustainable energy source, as long as the helium concentration in the atmosphere remains roughly constant, i.e. crustal emission and "anthropogenic" losses balance the outflow into interplanetary space.

\section{Acknowledgements}

The authors wish to thank D. Biskamp, L. Boccaccini, U. Fischer, F. Franza, S. Körner, W. Nuttall, L. Serio and M. Wanner for very helpful discussions. Dr. Wanner kindly checked the estimates of the energy required to replace the net loss of helium by extracting it from air. Dr. Clarke's very useful comments on our estimates of this quantity led us to make a small (upwards) correction in the proof stage. Moreover, he rightly points out that the helium inventory, or in-use stock, for 2760 fusion power plants $\left(1.7 \times 10^{5} \mathrm{t}\right)$ has to be acquired over time and that this is a point of some significance from a fusion policy point of view.

\section{References}

1. M. D. Garvey, Tight supply reins in the worldwide helium market, CryoGas Int. October 2011, p. 30.

2. E. Cook, The helium question, Science 206 (1979) 1141 .

3. A. M. Bradshaw and T. Hamacher, Non-regenerative natural resources in a sustainable system of energy supply, ChemSusChem 5 (2012) 550.

4. A. M. Bradshaw, T. Hamacher and U. Fischer, Is nuclear fusion a sustainable energy form? Fusion Eng. Des. 86 (2011) 2770.

5. W. J. Nuttall, R. H. Clarke and B. A. Glowacki (eds.), The Future of Helium as a Natural Resource, Routledge, Abingdon and New York, 2012.

6. W. J. Nuttall, R. H. Clarke and B. A. Glowacki, Stop squandering helium, Nature 485 (2012) 573.

7. R. H. Clarke and Z. Cai, Helium and fusion energy, in Ref. 6,p. 235.

8. E. g. M. A. Cook, Where is the Earth's radiogenic helium? Nature 179 (1957) 213.

9. W. I. Axford, The polar wind and the terrestrial helium budget, J. Geophys. Res., Space Phys. 73 (1968) 6855.

10. M. André and A. Yau, Theories and observations of ion energization and outflow in the high latitude magnetosphere, Space Sci. Rev. 80 (1997) 27.

11. Mineral Commodity Summaries, US Geological Survey, Reston, Virginia (2012).

12. V. Golubev et al, Development indices: Helium can be a motor for the Russian economy (in Russian), Gazprom In-house Journal, March (2012), p. 20.

13. R. U. Ayres, On the practical limits to substitution, Ecological Economics 61 (2007) 115.

14. M. C. Krupka and E. F. Hammel, Energy, helium and the future-II, Third Int. Conf. on Alternative Energy Sources, Miami Beach (1980). 
http://library.lanl.gov/cgi-bin/getfile?00237166.pdf

15. R. H. Clarke and R. Clare, Helium from the air, in Ref. 6, p. 118.

16. E.g. J. Schrier, Helium separation using porous graphene membranes, J. Phys. Chem. Lett 1 (2011) 2284.

17. Report of the Committee on Understanding the Impact of Selling the Helium Reserve, National Research Council of the National Academies, Washington, 2010.

18. L. Theiss, The US Government's role in providing helium to worldwide markets, CryoGas International, October 2007, p. 32.

19. L. Serio, personal communication

20. D. Maisonnier, European DEMO design and maintenance strategy, Fusion Eng. Des. 83 (2008) 858.

21. L. V. Boccaccini et al, Present status of the conceptual design of the EU test blanket systems, Fusion Eng. Des. 86 (2011) 478.

22. L. A. Sedano, Tritium cycle design for He-cooled blankets for DEMO, EFDA Report, TW4-TTB-COMPU, Garching, Germany (2006).

23. O.Gastaldi et al, Tritium transfers and main operating parameters impact for demo lithium-lead breeding blanket (HCLL) Fusion Eng. Des. 83 (2008) 1340.

24. L. Boccaccini and F. Franza, personal communication

25. L. Berton, Helium and tritium leaks in the PPCS BKT \& DV cooling circuits, CEA Technical Document DTN/STP/LTCG/2004/032 (2004)

26. U. Fischer, personal communication 